\title{
The electrophysiologic basis for lesions of the contemporary Maze operation
}

James L. Cox, MD, S. Chris Malaisrie, MD, Olga N. Kislitsina, MD, PhD, and Patrick M. McCarthy, MD

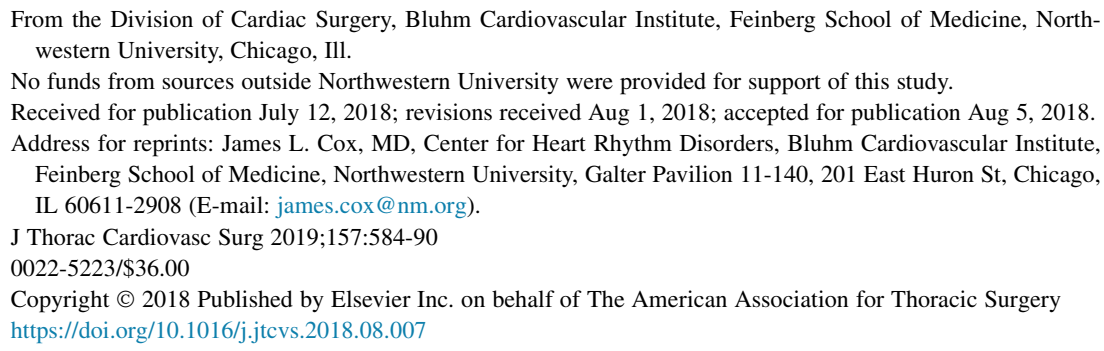

From the Division of Cardiac Surgery, Bluhm Cardiovascular Institute, Feinberg School of Medicine, Northwestern University, Chicago, Ill.

No funds from sources outside Northwestern University were provided for support of this study.

Received for publication July 12, 2018; revisions received Aug 1, 2018; accepted for publication Aug 5, 2018. Address for reprints: James L. Cox, MD, Center for Heart Rhythm Disorders, Bluhm Cardiovascular Institute,

Feinberg School of Medicine, Northwestern University, Galter Pavilion 11-140, 201 East Huron St, Chicago, IL 60611-2908 (E-mail: james.cox@nm.org).

J Thorac Cardiovasc Surg 2019;157:584-90

$0022-5223 / \$ 36.00$

Copyright $₫ 2018$ Published by Elsevier Inc. on behalf of The American Association for Thoracic Surgery https://doi.org/10.1016/j.jtcvs.2018.08.007

The specific pattern of atrial lesions has undergone several modifications since the first Maze procedure (Maze I) was performed on September 25, 1987. ${ }^{1}$ Modifications that have adhered to the concept of a maze pattern have proven to be as effective as the original maze pattern and include the historical Maze II procedure and the contemporary Maze III and Maze IV procedures. ${ }^{2,3}$ However, modifications that have not adhered to the maze concept have been less effective and include pulmonary vein isolation (PVI) alone, ${ }^{4}$ left-sided lesions alone, ${ }^{5}$ the Wolf "mini-Maze" procedure, ${ }^{6}$ the Dallas lesion set, ${ }^{7}$ the "5box Maze," 8 and many other less well-defined, arbitrary patterns. In addition, lesions based on modern, real-time maps have continued to be less successful than anatomybased Maze procedures because the specific macroreentry patterns during atrial fibrillation (AF) on one day may not be the same on the following day. ${ }^{9,10}$

The most common energy sources used to ablate AF surgically are radiofrequency (RF) energy and cryothermia (cryosurgery). Both types of bipolar RF clamps that are available to surgeons are excellent for creating permanent, transmural lesions, but not all critical parts of the atria are accessible to these clamps. For example, it is not possible to reach the left fibrous trigone with a bipolar clamp when performing the anterior left atrial lesion of the "Dallas" lesion set because the active portion of the clamps does not extend all the way to the tip of the clamps. This means that a cryolesion or unipolar RF must be added to the distal end of this lesion and to the right atrial lesion that extends to the tricuspid annulus. Cryosurgery is recommended to ablate the coronary sinus (CS), although in patients with dominant left coronary artery anatomy (90\%), a bipolar clamp can be placed across the so-called watershed area of the left atrioventricular (AV) groove, where there is no coronary artery, to create the mitral line and the CS lesion simultaneously. The problem with this approach is that the mitral line will not reach the mitral annulus or the RF can injure the posterior mitral valve leaflet because the

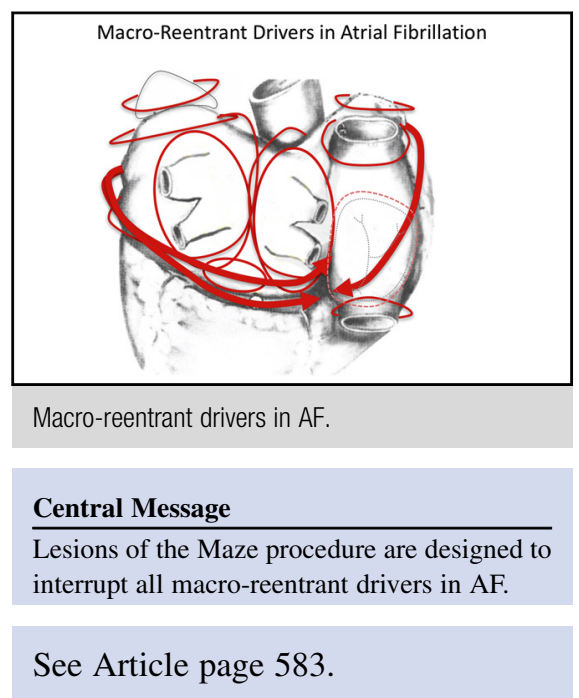

clamp tip extends across the annulus. If the anterior lesion of the "Dallas" lesion set or the mitral line of the Maze procedure is incomplete, these partial lines can result in peri-mitral atrial flutter. If the right atrial tricuspid line is incomplete, the patient can have classic right atrial flutter because of macro-reentry around the tricuspid valve annulus. Thus, if only 1 energy source is available for surgical AF ablation within a given institution, that energy source should be cryothermia because it is impossible to perform a complete Maze procedure reliably without using cryosurgery. Both cryosurgical devices that are available on the market are excellent, provided the surgeon knows how to use them.

It is the purpose of this article to clarify the currently recommended lesions that are designed to interrupt all of the known macro-reentrant circuits that have been shown to be responsible for sustaining AF. This recommended pattern of lesions differs from the Maze IV lesions first described by Damiano and colleagues $^{3}$ in 2002 only in the method by which the left atrial appendage is closed and in the positioning of 1 of the 3 lesions in the right atrium (RA).

\section{LEFT ATRIAL LESIONS}

The Maze procedure requires a total of only 7 lesions in the left atrium (LA), including placement of an epicardial clip at the base of the LA appendage (LAA). The recommended sequence in the performance of the LA lesions is as follows:

\section{Lesion 1: Isolation of the Left Pulmonary Veins}

Before isolating the left pulmonary veins, the ligament of Marshall is identified and divided with an electrocautery. It 

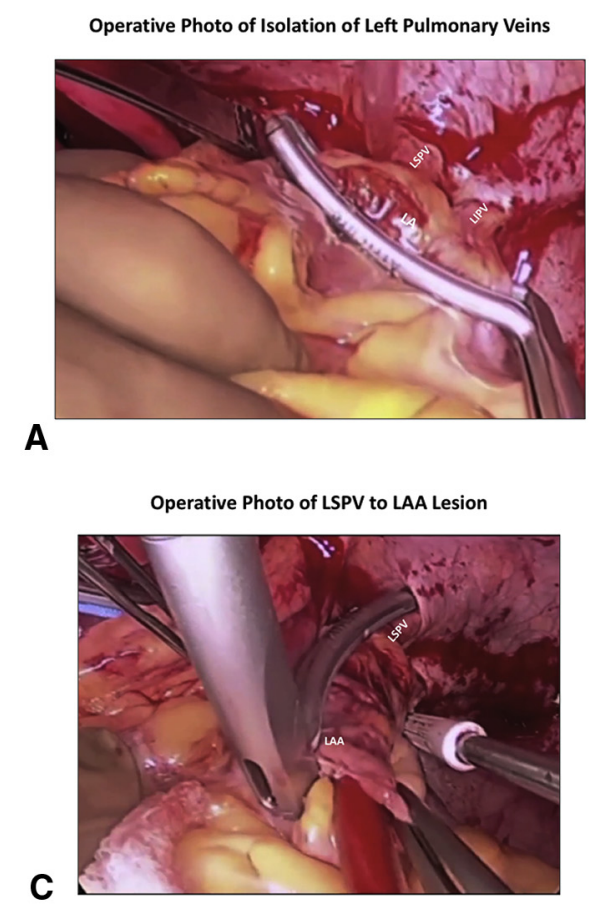

Drivers Remaining After Isolation of Left Pulmonary Veins

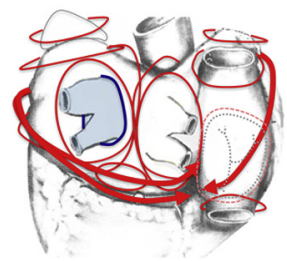

B

Drivers Remaining After LSPV to LAA Lesion

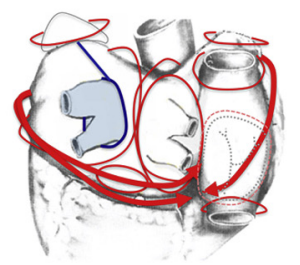

FIGURE 1. A, While exposing the left PVs, the ligament of Marshall is easily identified and should be divided with an electrocautery. The left PVs are then encircled by blunt dissection. The left PVs are isolated as a pair using a bipolar RF clamp. The bipolar RF clamp is placed well up onto the LA to avoid later PV stenosis. B, The red circles show the locations of the macro-reentrant drivers that have been mapped during AF. The 3 circuits that encircle the tricuspid valve, the anterior SVC orifice, and the posterior SVC orifice cause classic atrial flutter. However, if any one of them occurs in combination with one other circuit in the LA or RA, AF ensues. It is apparent from this figure why isolation of the left PVs alone has little or no effect on LSPAF because it does not interrupt any of the known macro-reentrant drivers that sustain AF. C, The lesion from the tip of the LAA to the left superior pulmonary vein (LSPV) crosses the so-called "Coumadin ridge" that is created by the in-folding of the lateral LA because of the presence of the ligament of Marshall. This lesion extends from the tip of the LAA well into the LSPV. The LAA portion of this lesion is unimportant because the entire LAA will be isolated and closed later. However, placing this lesion through the LAA tip and into the LSPV is technically easier than other methods for blocking conduction across the Coumadin ridge. This operative photograph shows how 1 arm of the bipolar RF clamp is placed inside and one outside the LAA and the distal end of the clamp is placed well into the LSPV to ensure that the Coumadin ridge has been crossed completely. D, The macro-reentrant AF driver that uses the Coumadin ridge and is interrupted by this lesion is a common cause of failure after catheter or surgical ablation of AF. $L S P V$, Left superior pulmonary vein; $L A A$, left atrial appendage.

is easily identified by retracting the heart to the right to expose the left pulmonary veins. The left pulmonary veins are then encircled and isolated as a pair using a bipolar RF clamp (Figure 1, A). It is important to place the bipolar RF clamp well up onto the LA itself away from the orifices of the left pulmonary veins to avoid later pulmonary vein stenosis.

Left PVI alone has little or no effect on persistent or longstanding persistent atrial fibrillation (LSPAF) because it does not interrupt any of the known macro-reentrant drivers responsible for sustaining $\mathrm{AF}$ (Figure 1,B). However, because left PVI is an integral part of a maze pattern of lesions, it is an essential step in the overall procedure.

\section{Lesion 2: Left Superior Pulmonary Vein to Left Atrial Appendage Lesion}

A lesion is created from the tip of the LAA well into the left superior pulmonary vein by placing $1 \mathrm{arm}$ of a bipolar RF clamp through a purse-string suture or a small incision in the tip of the LAA (Figure 1,C). This lesion interrupts potential macro-reentrant drivers that use the so-called "coumadin ridge" between these 2 structures (Figure 1,D).

\section{Lesion 3: Closure of the Left Atrial Appendage}

There are multiple ways to close the LAA during surgery. It can be amputated, but thrombosis of the suture line postoperatively is not uncommon after LAA amputation. ${ }^{11}$ Some prefer to close it with a stapling device, but the first randomized, control trial of this technique showed that stapling the LAA leaves residual pouches that can be thrombogenic and that epicardial suture closure fails to close the LAA completely. ${ }^{12}$ Murashita and colleagues ${ }^{13}$ reported excellent long-term follow-up results with their longitudinal endocardial double-layer plication of the LAA orifice. We prefer to close the LAA with an AtriClip (Atricure, Inc, Mason, Ohio), which not only accomplishes complete closure reliably ${ }^{14}$ but also electrically isolates the LAA myocardium. ${ }^{15}$ Nevertheless, there is an obvious 
Operative Photo of LAA Clip

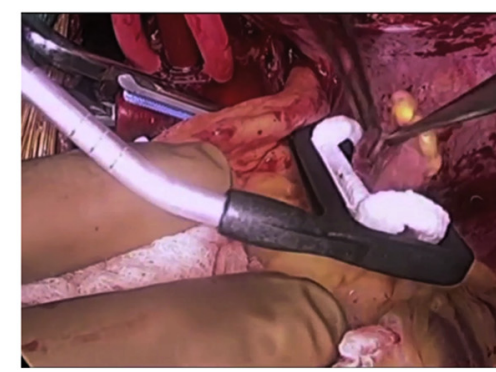

A

Operative Photo of Isolation of Right Pulmonary Veins

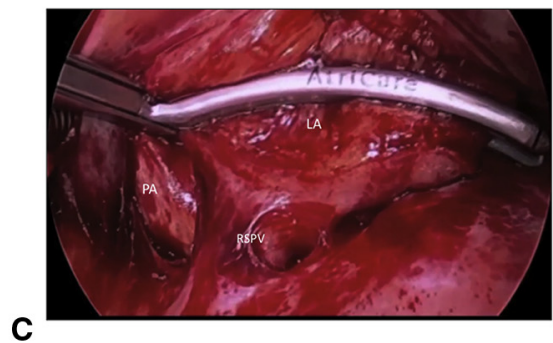

Drivers Remaining After LAA Clip

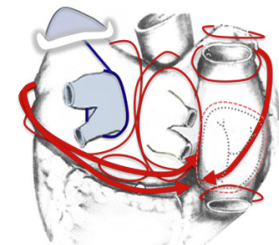

B

Drivers Remaining After Isolation of Right Pulmonary Veins

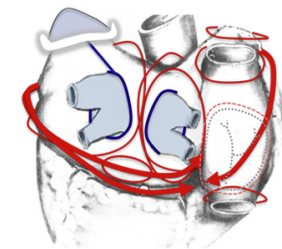

D

FIGURE 2. A, Clipping of the LAA is easily accomplished without changing the exposure after completion of the previous atrial lesions. B, Electrical isolation of the LAA eliminates triggers and macro-reentrant drivers that can reside within the LAA and therefore improves the results of AF ablation procedures. C, The right PVs are also isolated as a pair using a bipolar RF clamp. Waterston's groove is fully developed, and the right PVs are encircled using blunt dissection. The plane of dissection superiorly is between the right superior pulmonary vein and the right pulmonary artery. The bipolar RF clamp is placed well up onto the LA to prevent pulmonary vein stenosis. D, Like left PVI, right PVI has little effect on the macro-reentrant drivers responsible for sustaining persistent AF and LSPAF. LAA, Left atrial appendage.

advantage in cost savings of LAA amputation over device closure.

\section{Lesion 4: Isolation of the Right Pulmonary Veins}

Waterston's groove is developed fully by blunt dissection to expose the posterior-medial wall of the LA. A plane of dissection is established between the right superior pulmonary vein and the right pulmonary artery to expose the transverse sinus. The oblique sinus is opened below the inferior vena cava. The right pulmonary veins are then dissected free of any remaining nearby tissues and isolated by placing a bipolar RF clamp around them well up onto the LA to avoid later pulmonary vein stenosis (Figure 2, C). Again, this right PVI lesion alone does not significantly affect the macro-reentrant drivers that are responsible for sustaining persistent AF or LSPAF (Figure 2,D) but is an integral part of the overall maze lesion pattern. After isolation of the right pulmonary veins, the heart is arrested with cardioplegia and the LA is opened through a standard incision in Waterston's groove.

\section{Lesion 5: Creation of a Box Lesion}

Separate isolation of the right and left pulmonary veins as pairs is converted to a so-called box lesion around all 4 pulmonary veins and the posterior wall of the LA by placing "roof" and "floor" connecting lesions between the 2
(Figure 3, A). The 2 connecting lesions can be performed with bipolar RF clamps or linear cryoprobes. Because of several potential macro-reentrant drivers that use the posterior LA wall during persistent AF and LSPAF, these lesions have a significant impact on the overall success rates of surgical intervention (Figure 3, B).

\section{Lesion 6: Coronary Sinus Lesion}

The combination of LA lesions 1 to 5 will stop much of the AF that accompanies primary left-heart problems such as ischemic heart disease, mitral or aortic valve disease, left ventricular failure, and systemic hypertension. However, PVI can cause an iatrogenic arrhythmia called "peri-mitral atrial flutter," which occurs after PVI in approximately $10 \%$ to $15 \%$ of patients. Peri-mitral flutter is invariably less well tolerated than AF itself, and there are 2 theoretical ways of avoiding it. One way is to block all conduction across the left atrial isthmus between the mitral valve annulus and the inferior pulmonary veins, which will be described next. The other way is to block conduction across the anterior LA by placing a lesion from the superior portion of the box lesion anteriorly down to the left fibrous trigone, a part of the so-called Dallas lesion set. ${ }^{7}$ However, this anterior line frequently fails because it is difficult to create a fully transmural lesion in this area because of the thickness of Bachmann's bundle, and it is also hard to reach all the 
Operative Photo of Creation of Box Lesion

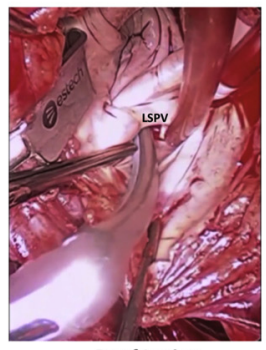

A

Roof Lesion

Operative Photo of CS Lesion

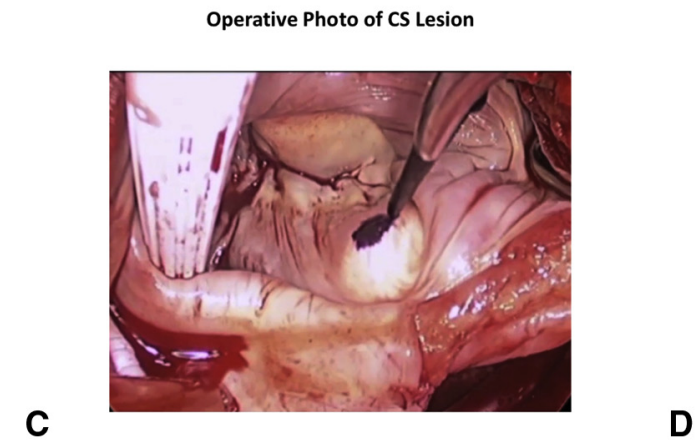

Drivers Remaining After Creation of Box Lesion

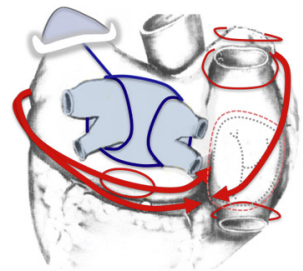

B

C

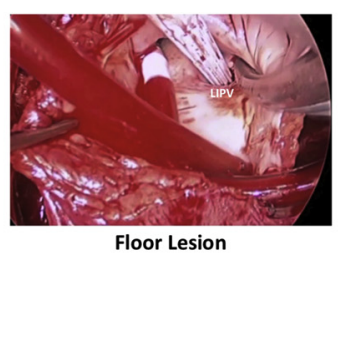

Drivers Remaining After CS Lesion

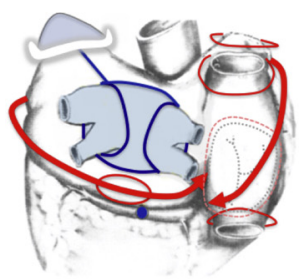

FIGURE 3. A, Box lesion isolates the posterior wall of the LA that can play an integral part in sustaining persistent and long-standing persistent AF by serving as a conduit for macro-reentrant drivers between the 2 pairs of PVs. The roof and floor lesions between the $2 \mathrm{PV}$ isolation lesions are created under cardioplegic arrest with a bipolar RF clamp or a linear cryoprobe. The roof lesion extends from the superior end of the left atriotomy to the LSPV. The floor lesion extends from the inferior end of the left atriotomy to the left inferior pulmonary vein. B, Completion of the box lesion results in the interruption of several macro-reentrant drivers responsible for persistent AF and LSPAF. C, The CS lesion is one half of the 2 lesions that are necessary to block conduction across the LA isthmus between the posterior mitral valve annulus and the inferior pulmonary veins. Macro-reentry using the LA isthmus is responsible for postoperative peri-mitral left atrial flutter (also called "atypical atrial flutter"), the most common mode of failure of the Maze procedure. A cryoprobe is placed behind the lower end of the left atriotomy with its tip directly against the epicardial surface of the CS. As the CS is frozen, an ice-ball appears on the inside (endocardial surface) of the LA, indicating that the CS lesion is transmural circumferentially. Because the ice-ball will disappear when the cryothermia is terminated, methylene blue dye is used to mark the exact site of the ice-ball during its formation. This is mandatory so that the surgeon will know exactly where to place the mitral line later because it is essential to place the mitral line and the CS lesion in the same plane. C, The CS lesion alone interrupts 1 of the 2 possible pathways of conduction across the LA isthmus, the CS itself. The other possible pathway of conduction is across the myocardium of the posterior-inferior LA myocardium of the LA isthmus and will be blocked with the mitral line. D, After creation of the CS lesion, the only left atrial macroreentrant circuits that have been documented are one confined to the LA isthmus and one participating in peri-mitral atrial flutter with conduction through the myocardium of the LA isthmus. These 2 circuits are the reason that a "mitral line" is necessary in addition to the CS lesion. CS, Coronary sinus.

way down to the fibrous trigone. Because incomplete lesions are proarrhythmic, we prefer the posterior approach directed at blocking conduction across the LA isthmus.

Because electrical conduction can occur across the LA isthmus not only in the atrial myocardium but also in the walls of the CS, it is necessary to create 2 separate, contiguous ablations to block conduction across the LA isthmus:

1. A "mitral line" in the atrial myocardium from the inferior part of the box lesion to the posterior mitral annulus.

2. A lesion in the CS in the same plane as the "mitral line."

Because the mitral line and CS lesions must be placed in the same plane to be effective, we prefer to place the CS lesion before creating the mitral line. The CS is easy to identify on the epicardial surface of the heart just behind and below the inferior end of the left atriotomy. The tip of a slightly curved cryoprobe is placed directly against the epicardial surface of the CS and pressed against the atrial side of the CS to completely collapse it against the epicardium of the LA. With the cryoprobe in position and adherent to the CS because of the initial freeze, attention is then directed to the endocardial surface of the LA directly overlying the position of the cryoprobe tip. An ice-ball will appear inside the LA, indicating circumferential ablation of the CS at that location. Although the endocardial ice-ball is visible, it is marked on the LA endocardium with methylene blue dye so that the exact position of the CS lesion can still be identified after the cryoprobe is turned off and the endocardial ice-ball has disappeared (Figure 3, $C$ ).

The CS lesion alone does not prevent peri-mitral flutter but does so only in combination with a mitral line in the same plane (Figure 3,D). Marking the site of CS ablation 
Operative Photos of Mitral Line

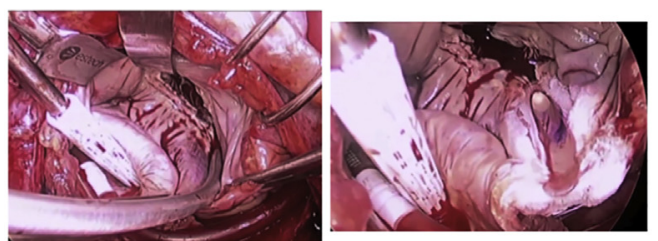

A

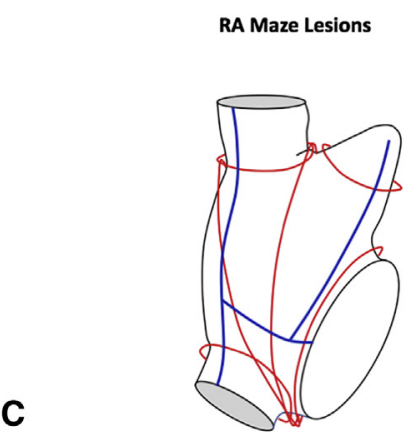

\section{C}

Drivers Remaining After Mitral Line

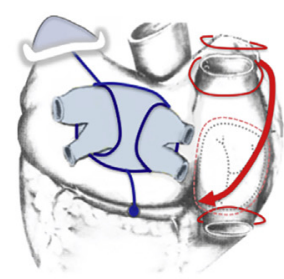

B

FIGURE 4. A, The mitral line is created in combination with the CS lesion to block conduction across the LA isthmus, thereby preventing the possibility of developing peri-mitral flutter postoperatively. A linear cryoprobe is placed precisely from the lower end of the left atriotomy, across the methylene blue mark, to the level of the mitral valve annulus. It is absolutely critical to place the mitral line in the same plane as that of the CS lesion to insure complete bidirectional block of conduction across the LA isthmus. B, The combination of the CS lesion and the mitral line prevents postoperative peri-mitral flutter. This completes all of the LA lesions of the Maze procedure. C, The red circles represent the macro-reentrant AF drivers that have been mapped in the RA during AF, and the blue lines represent surgical lesions. The 3 circuits that encircle the tricuspid valve, the anterior SVC orifice, and the posterior SVC orifice are those that cause classic atrial flutter. Note that all 3 flutter circuits and all but 2 of the fibrillation drivers use the CTI between the inferior vena cava orifice and the tricuspid valve annulus. The SVC inferior vena cava intercaval lesion interrupts some of these drivers but not all of them. This lesion alone will not ablate RA flutter or fibrillation. The combination of the " $\mathrm{T}$ " lesion and the intercaval lesion will ablate all classic atrial flutter. However, the possibility of reentry around the base of the RA appendage remains. To ablate the one remaining potential reentrant driver, a lesion is created from the anterior epicardial extent of the " $\mathrm{T}$ " lesion up to the tip of the RA appendage. D, These 3 RA lesions eliminate the possibility of RA drivers from sustaining atrial flutter, persistent $\mathrm{AF}$, or long-standing persistent $\mathrm{AF}$. $R A$, Right atrial.

inside the LA provides a guide for subsequent creation of the mitral line in the same plane as the CS lesion.

\section{Lesion 7: Mitral Line}

A linear cryoprobe is placed from the inferior end of the left atriotomy, directly across the marked location of the previous endocardial ice-ball, down to the level of the mitral valve annulus to create the mitral line in the same plane as the CS lesion (Figure 4, A). Like the other left atrial lesions, cryothermia is continued for 2 full minutes when the heart is under cardioplegic arrest.

These 7 lesions complete the lesion set in the LA (Figure 4, B). In AF that occurs secondary to left-heart disease, that is, "concomitant AF," these LA lesions alone are successful in ablating the majority of AF, although not equal to the results one can attain with a complete, biatrial Maze procedure. ${ }^{16}$ Deletion of the RA lesions in patients with concomitant AF associated with primary coronary artery, mitral valve, or aortic valve operations introduces an obligatory failure rate of between $10 \%$ and $30 \%$ depending on the duration of the AF and on performing the Maze procedure properly. ${ }^{16,17}$ Adding the RA lesions in the correct manner eliminates that obligatory failure rate and does not cause the patient to need permanent pacemakers more often postoperatively except by curing the $\mathrm{AF}$ and unmasking a sick sinus syndrome (SSS). ${ }^{18}$

\section{RIGHT ATRIAL LESIONS}

Although it is customary to think of the LA and RA as being separate structures because they belong to different isolated circulatory systems, they are one continuum of muscle electrically, and they are both involved in AF. It is impossible to create a maze pattern in the atrial continuum without including lesions in the RA. The literature has consistently shown that elimination of the RA lesions results in poorer outcomes than can be attained with biatrial lesions. ${ }^{16,17,19}$ The only exceptions to that observation have occurred in patients in whom the left atrial lesions, 
the right atrial lesions, or both were performed incorrectly or the individual lesions themselves were not transmural and contiguous.

Concern that the RA lesions cause an increased need for permanent pacemakers is the result of improperly placed RA lesions or misinterpretation of data. ${ }^{17-20}$ In our own series of patients from the 1980 s and 1990 s, ${ }^{21-23} 114$ patients were documented to have normal sinus node function preoperatively and not one of them required a pacemaker after the Maze procedure. Approximately 5\% of patients with paroxysmal AF have SSS preoperatively and will require a pacemaker after a Maze procedure. Approximately $10 \%$ of patients with persistent AF have SSS preoperatively and will require a pacemaker after surgery because of the "unmasking" of the SSS. Thus, the more successful AF ablation is, the higher the postoperative pacemaker requirements will be. ${ }^{17,18}$

In 2009, Santucci and colleagues ${ }^{24}$ showed the 3 circuits that are responsible for typical atrial flutter in the RA. Typical atrial flutter results from a single macro-reentrant circuit that always uses the cavo-tricuspid isthmus (CTI) (Figure $4, C$ ). The 3 variants are from the CTI around (1) the superior vena cava (SVC) orifice anteriorly, (2) the SVC orifice posteriorly, and (3) the tricuspid annulus.

Only 3 other macro-reentrant circuits have been documented in $\mathrm{AF}$, one around the orifice of the SVC, one around the orifice of the inferior vena cava, and one around the base of the RA appendage. To ablate all of these circuits, it is necessary to create only 3 lesions in the RA (Figure 4, C).

\section{Lesion 1: Superior Vena Cava to Inferior Vena Cava Lesion}

This is a lesion from the orifice of the SVC to the orifice of the inferior vena cava. This intercaval lesion alone has essentially no impact on atrial flutter or AF, so adding it to the LA lesions accomplishes nothing by itself.

\section{Lesion 2: Right Atrial “T” Lesion}

The combination of the SVC inferior vena cava lesion and the "T-lesion" prevents all typical RA atrial flutter and most AF. This lesion is anchored low on the intercaval lesion and extends distally to the level of the tricuspid annulus. The anchoring point of the T-lesion on the intercaval lesion should be two thirds of the way down from the orifice of the SVC. The RA muscle fibers in this region are diagonal, and the T-lesion should be placed in parallel with those fibers, that is, in a diagonal, not a horizontal, direction toward the right $\mathrm{AV}$ groove. When the $\mathrm{AV}$ groove is reached, the remainder of the T-lesion must be extended down to the tricuspid valve annulus from the inside of the RA. The only way to be certain of completion of the " $T$ " lesion to the true tricuspid valve annulus is with a cryoprobe.
When the T-lesion is added to the intercaval lesion, a CTI "flutter lesion" should never be performed because doing so isolates the lower one third of the RA from the rest of the heart and interferes with the ability to develop normal sinus bradycardia. ${ }^{18}$

\section{Lesion 3: Right Atrial Appendage Lesion}

After completing the intercaval and T-lesions, the only place in the RA where a macro-reentrant circuit has been documented is around the base of the RA appendage. There are 2 ways to preclude the development of this macroreentrant driver. One way is to place a lesion from the tip of the RA appendage medially down to the level of the tricuspid valve annulus, the so-called "10 o'clock lesion" or "counter-lesion," which was recommended in the Maze II, Maze III, and Maze IV procedures. Unfortunately, many surgeons were uncomfortable creating this particular lesion because they believed it to be too close to the AV node and worried about creating heart block. Because the only reason this lesion was introduced with the Maze II procedure and retained in the Maze III and Maze IV procedures was to prevent macro-reentry around the base of the RA appendage, ${ }^{2,3,25}$ it was suggested that the same goal could be realized by moving the lesion to a more readily accessible location on the RA free-wall (Figure 4, $C$ and $D)$. Cheema and colleagues ${ }^{26}$ have performed more than 100 Maze IV procedures using this modification of the RA lesions without any change in the success rate or pacemaker requirements compared with the previous " 10 o'clock" lesion of the Maze III and Maze IV procedures. The only important caveat is that this lesion must be placed as anteriorly as possible on the RA free-wall to avoid injury to the atrial pacemaker complex. ${ }^{18,27}$

These 3 RA lesions are safe and simple to perform and can be completed in 10 to 15 minutes. All of them can be created after the aortic crossclamp has been released during the warm reperfusion period before weaning from cardiopulmonary bypass. This precludes any added pump time or crossclamp time, so the length of the operation is not increased by the addition of the RA lesions. All 3 RA lesions can be created through 2 separate purse-string sutures as originally described in $2000^{28}$ or by opening the RA for creation under direct vision as illustrated in Figure 4, $C$ and $D$.

\section{Conflict of Interest Statement}

J.L.C. reports personal fees from Atricure, Adagio Medical, SentreHEART, PAVmed, and Northwestern University. P.M.M. reports personal fees from Atricure and Edwards Lifesciences. All other authors have nothing to disclose with regard to commercial support.

The authors wish to express their gratitude to Dr Marc Gerdisch of Franciscan St. Francis Health in Indianapolis, Indiana, for the 
use of the operative photographs that were taken by author J.L.C. during a Maze-IV procedure performed by Dr Gerdisch.

\section{References}

1. Cox JL. The first Maze procedure. J Thorac Cardiovasc Surg. 2011;141:1093-7.

2. Cox JL, Jaquiss RD, Schuessler RB, Boineau JP. Modification of the Maze procedure for atrial flutter and atrial fibrillation. II. Surgical technique of the Maze III procedure. J Thorac Cardiovasc Surg. 1995;110:485-95.

3. Damiano RJ Jr, Schwartz FH, Bailey MS, Maniar HS, Munfakh NA, Moon MR, et al. The Cox Maze IV procedure: predictors of late recurrence. J Thorac Cardiovasc Surg. 2011;141:113-21.

4. Oral H, Knight BP, Tada H, Ozaydin M, Chugh A, Hassan S, et al. Pulmonary vein isolation for paroxysmal and persistent atrial fibrillation. Circulation. 2002:105:1077-81

5. Sakamoto Y, Takakura H, Onoguchi K, Hachiya T, Sasaki T, Yoshitake M, et al. Cryosurgical left-sided Maze procedure in patients with valvular heart disease: medium-term results. Ann Thorac Cardiovasc Surg. 2011;17:148-52.

6. Wolf RK, Schneeberger EW, Osterday R, Miller D, Merrill W, Flege JB Jr, et al Video-assisted bilateral pulmonary vein isolation and left atrial appendage exclusion for atrial fibrillation. J Thorac Cardiovasc Surg. 2005;130:797-802.

7. Edgerton JR, Jackman WM, Mahoney C, Mack MJ. Totally thoracoscopic surgical ablation of persistent $\mathrm{AF}$ and long-standing persistent atrial fibrillation using the "Dallas" lesion set. Heart Rhythm. 2009;6(12 Suppl):S64-70.

8. Sirak J, Jones D, Schwartzman D. The five-box thoracoscopic Maze procedure Ann Thorac Surg. 2012;95:986-9.

9. Tilz RR, Rillig A, Thum AM, Arya A, Wohlmuth P, Metzner A, et al. Catheter ablation of long-standing persistent atrial fibrillation: 5-year outcomes of the Hamburg sequential ablation strategy. J Am Coll Cardiol. 2012;60:1921-9.

10. Weerasooriya R, Khairy P, Litalien J, Macle L, Hocini M, Sacher F, et al. Catheter ablation for atrial fibrillation: are results maintained at 5 years of follow-up? J Am Coll Cardiol. 2011:57:160-6.

11. Cullen MW, Stulak JM, Powell BD, White RD, Ammash NM, Nkomo VT. Left atrial appendage patency at cardioversion after surgical left atrial appendage intervention. Ann Thorac Surg. 2016;101:675-81.

12. Healey JS, Crystal D, Lamy A, Teoh K, Semelhago L, Hohnloser SH, et al. Results of a randomized controlled pilot study of left atrial appendage occlusion during coronary bypass surgery in patients at risk for stroke. Am Heart J. 2005;150:288-93.

13. Murashita T, Rankin S, Wei LM, Roberts HG, Alkhouli MA, Badhwar V. Oral anticoagulation may not be necessary for patients discharged in sinus rhythm after the Cox Maze IV procedure. J Thorac Cardiovasc Surg. 2018;155:997-1006.
14. Caliskan E, Cox JL, Holmes DR Jr, Meier B, Lakkireddy DJ, Falk V, et al. Interventional and surgical occlusion of the left atrial appendage. Nat Rev Card. 2017; $14: 727-43$

15. Yorgun H, Canpolat U, Kocyigit D, Coteli C, Evranos B, Aytemir K. Left atrial appendage isolation in addition to pulmonary vein isolation in persistent atrial fibrillation: one-year clinical outcomes after cryoballoon-based ablation. Europace. 2017; 19:758-68

16. Barnett SD, Ad N. Surgical ablation as treatment for the elimination of atrial fibrillation: a meta-analysis. J Thor Cardiovasc Surg. 2006;131: 1029-35.

17. Cox JL. Surgical ablation for atrial fibrillation. N Engl J Med. 2015;373:483.

18. Cox JL, Ad N, Churyla SC, Pham DT, Kruse J, Kislitsina ON, et al. The maze procedure and postoperative pacemakers. Ann Thorac Surg. June 5, 2018 [Epub ahead of print].

19. Ruaengsri C, Schill MR, Khiabani AJ, Schuessler RB, Melby SJ, Damiano RJ Jr. The Cox-maze IV procedure in its second decade: still the gold standard? Eur J Cardiothorac Surg. 2018;53:119-25.

20. Gillinov AM, Gelijns AC, Parides MK, DeRose JJ Jr, Moskowitz AJ, Voisine P, et al; for the CTSN Investigators. Surgical ablation of atrial fibrillation during mitral-valve surgery. N Engl J Med. 2015;372:1399-409.

21. Cox JL, Boineau JP, Schuessler RB, Kater KM, Lappas DG. Five-year experience with the Maze procedure for atrial fibrillation. Ann Thorac Surg. 1994;56:814-24.

22. Cox JL, Schuessler RB, Lappas DG, Boineau JP. An 8 1/2 year clinical experience with surgery for atrial fibrillation. Ann Surg. 1996;224:267-75.

23. Prasad S, Maniar H, Camilo C, Schuessler R, Boineau J, Sundt T, et al. The Cox maze III procedure for atrial fibrillation: long-term efficacy in patients undergoing lone versus concomitant procedures. J Thorac Cardiovasc Surg. 2003;126: 1822-8.

24. Santucci PA, Varma N, Cytron J, Akar JG, Wilber DJ, Al Chekakie MO, et al. Electroanatomic mapping of postpacing intervals clarifies the complete active circuit and variants in atrial flutter. Heart Rhythm. 2009;6:1586-95.

25. Cox JL, Boineau JP, Schuessler RB, Lappas DG. Modification of the Maze procedure for atrial flutter and atrial fibrillation. I-Rationale and surgical results. $J$ Thorac Cardiovasc Surg. 1995;110:473-84.

26. Cheema FH, Younus MA, Siddiqui OT, Younus MJ, Mahmood MA, Pervez MB, et al. Early results of the modified right atrial lesion set for the Cox-CryoMaze procedure. Innovations. 2016;11:342-8.

27. Boineau JP, Schuessler RB, Canavan TE, Corr PB, Cain ME, Cox JL. The human atrial pacemaker complex. J Electrocardiol. 1989;22(Suppl):189-97.

28. Cox JL. The minimally invasive Maze-III procedure. Op Tech Thorac Cardiovasc Surg. 2000;5:79-92. 